\title{
Perspective
}

PERSPECTIVE Actualité en histoire de l'art

$1 \mid 2012$

Art et pouvoir

\section{Arts, violences, identités : l'apport des études postcoloniales}

Art, violence, identity: postcolonial studies in the spotlight

Maureen Murphy, Zahia Rahmani, Todd Shepard, Elvan Zabunyan et Rémi Labrusse

Traducteur : Géraldine Bretault

\section{OpenEdition}

\section{Journals}

Édition électronique

URL : http://journals.openedition.org/perspective/518

DOI : $10.4000 /$ perspective. 518

ISSN : 2269-7721

Éditeur

Institut national d'histoire de l'art

Édition imprimée

Date de publication : 30 juin 2012

Pagination : 56-69

ISSN : $1777-7852$

Référence électronique

Maureen Murphy, Zahia Rahmani, Todd Shepard, Elvan Zabunyan et Rémi Labrusse, " Arts, violences, identités : l'apport des études postcoloniales », Perspective [En ligne], 1 | 2012, mis en ligne le 30

décembre 2013, consulté le 01 octobre 2020. URL : http://journals.openedition.org/perspective/518 ; DOI : https://doi.org/10.4000/perspective.518 


\title{
Arts, violences, identités : I'apport des études postcoloniales
}

\author{
Points de vue de Maureen Murphy, Zahia Rahmani, Todd Shepard et Elvan Zabunyan, \\ avec Rémi Labrusse
}

Les études postcoloniales n'ont fini de susciter ni les questions ni les controverses : signe de leur capacité, aujourd'hui comme hier, à inventer de nouvelles démarches et à ouvrir de nouveaux champs d'investigation. En reliant, à la suite d'Edward Said, I'analyse historique des situations coloniales à la notion plus large $d^{\prime}$ " empire », en s'articulant aussi aux études de genre, elles ont permis d'aborder différemment, au-delà de la question coloniale stricto sensu, les mouvements incessants de constitution et de déconstruction de l'individu, conflictuellement immergé dans les réseaux de pouvoirs qui s'exercent sur lui et en lui. C'est pourquoi l'approche postcoloniale peut entrelacer l'histoire à l'anthropologie, à la sémiotique, à la sociologie, à la psychanalyse, aux études littéraires - ou à l'histoire de l'art, dans la mesure où les images, après les textes, sont apparues comme des sources décisives de réflexion.

Depuis trente ans, en dépit de maintes résistances, le postcolonial s'immisce donc avec obstination dans le discours académique de I'histoire de l'art, y démultiplie les possibilités du regard, y encourage la prolifération de sources nouvelles, y fait entendre d'autres voix en stimulant les brouillages disciplinaires et y désigne les horizons éthiques et politiques de tout travail de recherche : la démarche analytique se double d'un engagement qui ne saurait être " purement " esthétique ; en retour, aucun engagement éthique ou politique ne peut se dérober aux exigences critiques d'une autoanalyse. Cette tension entre militantisme et pensée critique rapproche aujourd'hui, dans une perspective postcoloniale, le savoir et la création, l'exercice de la raison herméneutique et la production contemporaine d'œuvres visuelles ou littéraires.

Une puissante ouverture en résulte, géographique et discursive. Le champ des études et de la création, le réseau des échanges intellectuels s'élargissent au-delà des frontières de l'Occident. Les usages traditionnels du discours académique ou esthétique sont modifiés et animés par les contradictions mêmes sur lesquelles repose l'approche postcoloniale et qui lui donnent vie : contradictions entre la radicalité réaliste de l'action et la diffraction critique de la réflexion, entre l'affirmation du relativisme des identités et la reconnaissance de la légitimité des désirs d'authenticité, entre une mélancolie poststructuraliste et l'insistante résurgence de convictions humanistes. Encore faut-il s'inquiéter de savoir dans quelle
Rémi Labrusse, professeur à I'université Paris OuestNanterre, travaille notamment sur les rapports entre l'Europe et les arts non-occidentaux au XIXe siècle. II a récemment publié Islamophilies : I'Europe moderne et les arts de I'Islam (Paris, 2011).

Maître de conférences à I'université Paris 1, Maureen

Murphy étudie I'histoire de la réception et de la représentation des arts d'Afrique en occident. Elle est l'auteur de De l'imaginaire au musée : les arts $d^{\prime}$ Afrique à Paris et à New York (1936-2006) (Dijon, 2009).

Écrivain, Zahia Rahmani dirige le programme de I'INHA sur l'art dans la mondialisation et travaille sur I'historiographie des études postcoloniales. Elle a écrit « Le moderne comme point d'arrivée sans fin ", dans Qu'est-ce que le contemporain? (Paris, 2010).

Historien à la Johns Hopkins University, Todd Shepard est spécialiste de la France et de son empire colonial au XXe siècle. Il examine les interactions entre l'histoire de l'impérialisme, les institutions étatiques, et les identités nationales, raciales et sexuelles.

Elvan Zabunyan est maître de conférences à l'université Rennes 2. Ses recherches, sur l'art nord-américain depuis les années 1960, s'inscrivent dans les cultural studies, les théories postcoloniales et les études de genre. 
mesure l'énergie de la contradiction et les effets salutaires de l'indiscipline peuvent résister aux tendances à la fermeture académique, sur un plan universitaire, ou aux simplifications théoriques réclamées par les institutions de tous ordres - musées, médias ou marchés. [Rémi Labrusse]

Rémi Labrusse. Les études postcoloniales sont d'abord passées par l'étude des textes ; c'est par là, à l'origine, qu'ont été pensés les liens entre pouvoirs et représentations. Les arts visuels, tard venus dans ce champ d'analyse, ont-ils contribué à en modifier la dynamique intellectuelle?

Elvan Zabunyan. Dans le cas de la France, il me semble plus judicieux de parler dans un premier temps de " théories postcoloniales » plutôt que d' « études postcoloniales », dans la mesure où cette dernière appellation renvoie à une discipline universitaire qui est encore inexistante ici. Les domaines de recherche scientifiques qui incluent des perspectives postcoloniales ou qui s'adossent aux théories postcoloniales, à partir notamment de la figure tutélaire d'Edward Said, qui a posé dès 1978 un modèle de pensée inédit avec Orientalism ${ }^{1}$ (fig. 1), se trouvent principalement dans le champ de la littérature, de la philosophie, de la sociologie et de I'histoire. Les théories postcoloniales permettent de véhiculer des points de vue qui renversent les habituels paramètres de centre et de périphérie - avec l'Occident au centre et le reste du monde marginalisé.

Le texte, critique et/ou littéraire, a d'abord été la forme privilégiée en tant que prolongement logique des activités d'enseignement et de recherche. L'importance croissante des intellectuel-le-s issu-e-s de pays à la lourde histoire coloniale, qui ont occupé au tournant de la décennie 1990 une place prépondérante dans les universités en Grande-Bretagne (Homi Bhabha et Stuart Hall) et aux États-Unis (Edward Said et Gayatri Spivak), a radicalement transformé I'espace théorique et, par là même, a influé sur la circulation des informations par la diffusion de périodiques et de publications spécialisées. Ces dernières sont devenues des structures méthodologiques fondamentales pour toute personne orientant sa réflexion sur les problématiques littéraires, historiques, philosophiques ou artistiques, liées au postcolonialisme $^{2}$. Une exposition comme Magiciens de la Terre, organisée en 1989 au Musée national d'art moderne à Paris ${ }^{3}$ (fig. 2), est contemporaine de ces nouvelles orientations de la recherche liées aux déplacements culturels, et a donné une visibilité à des auteurs et à des artistes qui jusque-là n'en avaient pas.

Ce déplacement de paradigmes, qui est devenu l'enjeu majeur des rapports de pouvoirs, est sans doute le point central de l'influence des théories postcoloniales sur I'histoire de l'art : l'approche vise à renverser l'hégémonie d'une approche eurocentriste, et à accorder une valeur égale à des formes de création diverses par une diffusion à l'échelle mondiale. Cependant, d'un point de vue à la fois pratique, historique et théorique, l'exposition est soumise à des impératifs économiques et politiques, comme on l'a vu avec Magiciens de la terre; l'étude de l'exposition comme médium de diffusion est donc aussi un outil des plus intéressants pour saisir les effets de tels impératifs.

Maureen Murphy. L'exposition Primitivism in $20^{\text {th }}$ Century Art, organisée par William Rubin au Museum of Modern Art de New York en $1984^{4}$, marque un tour-

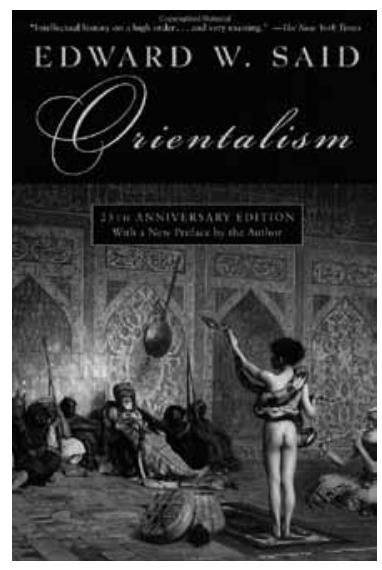

1. Edward Said, Orientalism, New York, 1978.

2. Vue de l'exposition Magiciens de la terre, Paris, Centre Georges Pompidou/La Grande halle de la Villette, 1989.

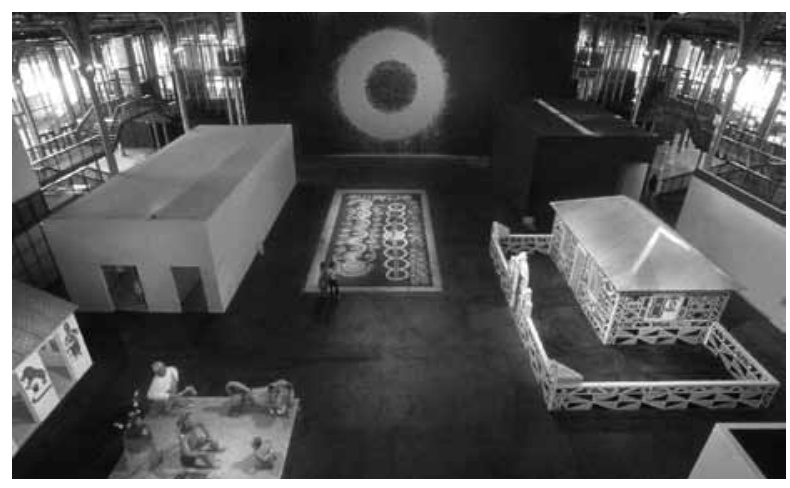




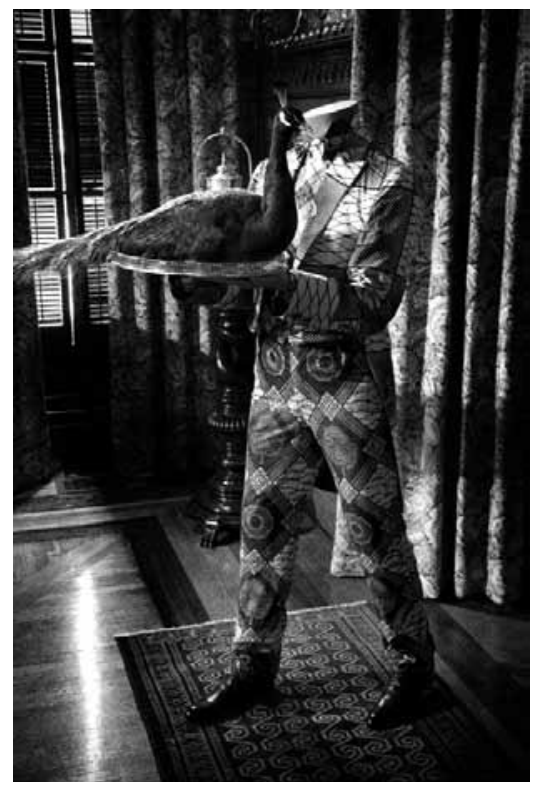

nant décisif dans les réflexions sur les rapports entre arts et pouvoirs. Le débat suscité par cette manifestation a provoqué une prise de conscience des enjeux politiques liés aux questions de représentation et a contribué à déconstruire l'idée d'une neutralité politique du modernisme en art. Aux États-Unis, une réflexion née dans le champ des études littéraires s'est alors étendue à celui de l'histoire de l'art et de la muséologie : les enjeux de pouvoir et de domination postcoloniaux furent pris en compte dans l'analyse des œuvres ainsi que dans les processus de monstration muséale. Primitivism in $20^{\text {th }}$ Century Art a constitué le paroxysme de la réappropriation formaliste des arts d'Afrique, des Amériques et d'Océanie, pris dans I'histoire longue de leur réception, et a marqué ainsi un véritable tournant (du moins aux États-Unis). Les vives critiques que l'événement a suscitées ${ }^{5}$ ont participé d'un mouvement plus vaste de décentrement du regard induit par les études postmodernes et postcoloniales. II devenait nécessaire, à l'époque, de donner la parole aux peuples opprimés, de les sortir de l'ombre dans laquelle l'Occident les avait longtemps maintenus.

Dans les années 1990, dans le champ de l'art contemporain, les conséquences de cette prise de conscience politique furent, à mon

3. Yinka Shonibare, Party Time: Re-Imagine America (détail), 2009. avis, de deux ordres. D'une part, une forme $d^{\prime}$ ' essentialisme stratégique » (pour reprendre l'expression de Gayatri Spivak ${ }^{6}$ ) s'est mise en place, tendant à promouvoir l'art de certaines parties du monde sous un angle identitaire et recourant donc à des catégories telles que l'art " africain », « indien », ou plus récemment "islamique ». D'autre part, et presque paradoxalement, la sensibilité induite par les études postcoloniales constitua, pour certains artistes, les outils d'un renversement de ces mêmes catégories identitaires. Un artiste comme Yinka Shonibare confronté dès ses premières années d'études artistiques à Londres aux attentes africanistes de son entourage, a décidé de mener un travail de déconstruction des notions d'authenticité et d'africanité. En réinventant notamment des scènes de grands maîtres du XVIII ${ }^{\mathrm{e}}$ siècle dans des installations ou "tableaux vivants " dans lesquels les personnages sont vêtus de tissus wax (textile associé spontanément à l'Afrique, mais originellement produit en Indonésie puis distribué par les Anglais et les Hollandais sur le continent africain), il déjoue les attentes du public pour questionner les termes de la construction identitaire (fig. 3). Ce détour par le siècle des Lumières correspond tout à fait aux logiques postcoloniales visant à questionner les liens entre passé et présent.

Zahia Rahmani. L'intelligence d'Edward Said réside dans la manière dont il s'est approprié le terme " orientalisme » pour le forger en concept ${ }^{7}$. Ce mouvement esthétique et littéraire en prise avec l'histoire coloniale britannique et française était tout à fait approprié pour servir de socle conceptuel. En convoquant et en reformulant le rapport Occident/Orient, Said déjoue la relation Nord/Sud pour introduire sur la scène de la critique le fait colonial dans sa relation au récit et, selon lui, à l'existence.

Dans le sillage de Said, les publications actuelles appellent une nouvelle méthode critique qui envisage le savoir à partir des territoires précoloniaux et extracoloniaux, et qui consiste en la saisie d'un contrechamp vu par l'autre en définition et en construction, comme acte essentiel de connaissance. Certains s'obstinent à faire un procès en essentialisme à cette position, car ils ne parviennent pas à la saisir. Rarement un nouveau champ d'études a donné lieu à une telle production bibliographique. On dénombre aujourd'hui sur le sujet plus de 5000 essais et quelque 
300 catalogues d'art. Une part importante de ces ouvrages est consacrée aux pratiques et aux biens culturels ainsi qu'à l'écriture de l'histoire, comme en témoigne la bibliographie sur l'art et la mondialisation que nous avons établie à l'Institut national d'histoire de $\mathrm{I}^{\prime} \mathrm{art}^{8}$. Ces publications relèvent de nombreux sous-groupes d'études tels que le genre, les traditions juridiques, le travail, l'agriculture, la pharmacopée, la religion, le textile, le vêtement, l'archéologie, l'architecture, la littérature ou l'image. Souvent ces champs interfèrent. Ils peuvent concerner un ensemble et ses diversités ou bien relever d'une mise en perspective précoloniale ou postcoloniale, comme par exemple entre les pratiques picturales des femmes indiennes d'Amérique du Nord avec celles des femmes berbères du Maghreb ou de l'Afrique subsaharienne. Cela permet de penser le commun et les transferts, la migration des formes, le déplacement des hommes mais aussi la distinction. Les théories postcoloniales se servent de l'ethnographie, et s'en éloignent tout autant, car elles doutent de l'idée d'une culture authentique.

Si c'est une lecture spécifique de la littérature dite " coloniale » qui a favorisé l'émergence des études postcoloniales, c'est aussi une certaine pratique de I'intertextualité propre à la littérature comparée qui a permis à ces études dans le monde anglo-saxon de s'étendre à tous les arts (le théâtre, la danse, le cinéma...). La critique postcoloniale intervient dans le champ des arts visuels et écarte de fait une approche strictement formelle de l'art. La modernité picturale " arabe » des années 1950 et 1960, par exemple, s'est constitué en lien avec le mouvement moderne européen mais aussi contre lui dans la mesure où elle a accompagné des mouvements de libération nationale (fig. 4). L'exposition Short Century a très bien montré cette ambiguïté ${ }^{9}$.

Dans les empires coloniaux modernes, un nombre important d'images relevant des différentes identités qu'ils comportaient a été produit et diffusé pour le public européen. Le postcolonial investit en "creux » la subjectivité, voire la politique, qui a accompagné la production et la diffusion de ces images. En Inde, on ne peut penser les théories postcoloniales en dehors de la réflexion sur la période qui a précédé la colonisation anglaise. L'Inde d'avant la colonisation doit-elle être abordée comme un territoire indien ou moghol ? Qu'a fait la colonisation anglaise de l'héritage moghol, de sa tradition architecturale et figurative et de ces recyclages ? Ces questions et bien d'autres sont alimentées par les études postcoloniales et relèvent plus spécifiquement des études subalternes. Le collectif de chercheurs qui a inauguré en Inde en 1982, dans la continuité des théories postcoloniales, les subaltern studies ${ }^{10}$, a ouvert la voie dans les pays dits du Sud à une critique postcoloniale de

4. Jawad Saleem, Monument à la liberté, 1959, Bagdad, place Tahrir. la modernité et de ses formes, introduisant les effets de rupture culturelle induits par les changements que met en place le colonialisme ${ }^{11}$.

Le modèle d'empire qui s'est exercé en Inde, en l'occurrence celui de la Grande-Bretagne, s'est constitué pour servir une conquête. L'historien de I'art Éric Michaud, dont les travaux ne semblent pas a priori relever de la théorie postcoloniale, a remarquablement décrit comment l'art et sa discipline théorique ont participé, par l'exercice de rénovation de l'histoire de l'art, de la constitution du matériau symbolique de cette conquête. Porté à son point d'acmé, le génie d'un peuple - sa création, son art, vus comme tels, et son identité - ne peut que vouloir marquer sa durée nou-

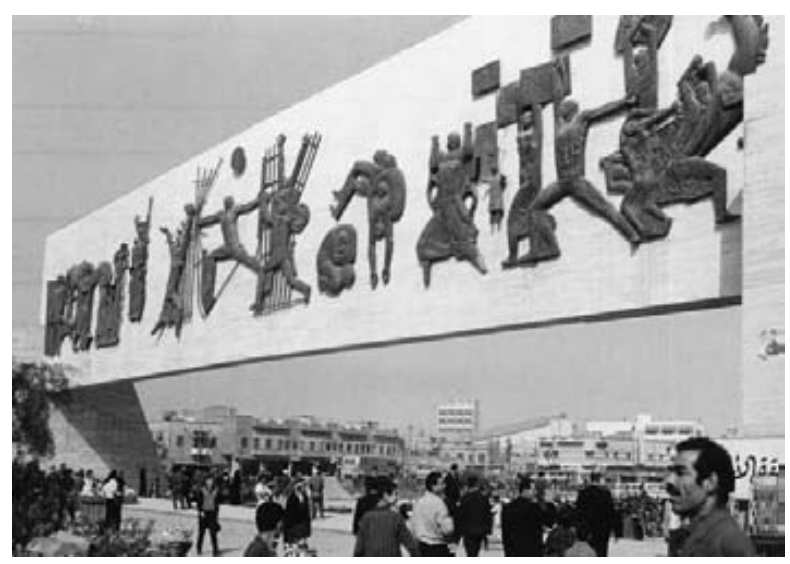


velle par une volonté de continuité et d'extension, qui passera nécessairement par la conquête $d^{\prime}$ un autre oblitéré et écarté, assujetti et allié à la cause, à des fins de pérennité, voire $d^{\prime}$ éternité ${ }^{12}$. Cela confirme la thèse $d^{\prime}$ Eric Hobsbawm et Terence Ranger, selon laquelle la tradition est un processus $d^{\prime}$ invention récente de légitimation ${ }^{13}$. Certains intellectuels ont saisi l'opportunité de cette dynamique comparatiste que l'on nomme aujourd'hui les "études visuelles ». Il faut d'ailleurs souligner que, sous cet intitulé, les études sur l'art sont enfin accueillies dans des pays qui jusque-là envisageaient l'histoire de l'art comme une discipline « ennemie » : on trouve un enseignement de visual studies à Accra, Cape Town, Dar es-Salaam, Delhi, Hong Kong, Lagos, Pékin, Otago, Shanghai et d'autres villes encore.

L'image, avec ses raisons et ses usages, est le support essentiel de ces études, car elle a servi de vecteur là où la langue de l'empire ne pouvait être lue. En Afrique mais aussi en Inde, plus de $90 \%$ des locuteurs ne lisaient pas cette langue. Or, tout message ayant besoin d'un médiateur, ce sont les images générées par cette médiation qui s'étudient aujourd'hui en Asie et en Afrique, et qui attirent de plus en plus d'étudiants en art, dont des Européens, vers ces régions.

Todd Shepard. Un des apports majeurs des études postcoloniales a été d'insister sur le rôle du projet impérial dans la constitution de nombreuses disciplines académiques. D'un point de vue historique, les empires ont été fondés et les raisons de cette fondation ont été élaborées au moment précis où le mode académique d'organisation de la recherche et du savoir était en voie de redéfinition. L'histoire de la colonisation a ainsi modelé les outils, les modes de classification et surtout certaines frontières précises en fonction desquelles les communautés de chercheurs définissent leurs objets d'étude et leurs méthodes d'analyse. Le défi épistémologique lancé par les études postcoloniales aux conceptions universalistes et, par conséquent, aux postulats fondateurs du savoir " moderne » contribue à expliquer l'immense résistance que les chercheurs ont eu tendance à opposer aux revendications postcoloniales. Cette résistance traduit aussi la nostalgie de la tour d'ivoire, d'un monde imaginaire de science pure que les penseurs du postcolonialisme, à la suite de Jacques Derrida, ont révélé comme étant une «mythologie blanche $»^{14}$.

Explorer avec sérieux les champs ouverts par les études postcoloniales nécessite de comprendre comment l'œuvre de chaque empire a modelé différemment les discussions théoriques et les disciplines et a contribué à définir leurs objets d'étude. Les stratégies adoptées par les chercheurs en littérature ne sont pas nécessairement préconisées pour les historiens ; les questions historiques liées à l'empire britannique ne coïncident pas forcément avec celles que pose le colonialisme français. Les différentes interprétations des textes peuvent inspirer mais ne devraient certainement pas déterminer la manière dont nous analysons les images. Le devoir du postcolonialisme consiste à approfondir ce qui est en jeu, les réseaux, les ressources et les hypothèses qui découlent de ces histoires singulières. Il faut aussi se rappeler que le recours à l'argument des divergences, notamment nationales, reste un bon moyen d'éviter de se confronter vraiment à ces questions.

En France, nombre d'historiens de l'art, à la suite des arguments proposés par l'anthropologue Jean-Loup Amselle ${ }^{15}$, se sont récemment engagés dans des discussions portant sur le postcolonialisme, avant tout pour défendre des conventions propres à leur discipline. Certains d'entre eux se sont focalisés sur l'importance regrettable que les discussions dans les médias ont accordée à des affirmations sans nuances et mal informées à propos des images " coloniales», notamment 
autour des interventions de Pascal Blanchard, qui propose une vision manichéenne et misérabiliste de ces images, réduisant tout à des déclinaisons du racisme au lieu d'examiner, par exemple, pourquoi des historiens de l'art qualifiés ont échoué à réaliser ce travail antérieurement. D'autres endossent un antiaméricanisme primaire pour refuser de se prononcer par rapport aux penseurs anglo-saxons du postcolonialisme et à leurs textes; ils affirment que les études postcoloniales sont assimilables aux « politiques identitaires » (américaines), qu'elles se contentent

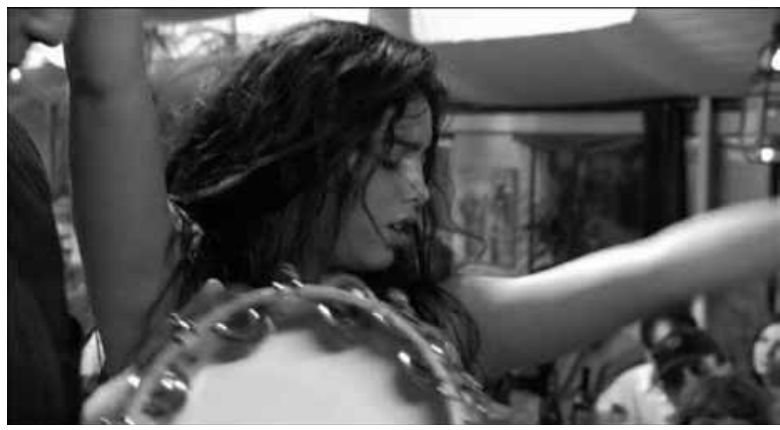
de s'incliner devant les «indigènes persécutés » et de les célébrer (alors que les penseurs du postcolonialisme aux États-Unis rejettent activement ces approches). Dans la lignée de l'anti-intellectualisme promu par le scandaleux article 4 de la loi du 23 février 2005 - qui vise à réduire les projets de recherche à de banales déclarations quant au rôle " positif » ou " négatif » du colonialisme - ou de l'affirmation tout aussi scandaleuse que I'histoire serait une science pure absolument distincte de la " politique » - ce que trop de chercheurs ont affirmé lorsqu'on les a interrogés au sujet des "lois mémorielles»-, le corporatisme disciplinaire l'a trop souvent emporté au détriment d'un examen rigoureux de ce que les historiens de l'art pourraient retirer des débats postcoloniaux ${ }^{16}$. Jusqu'à présent, seule une mince part des historiens de l'art français a mis son expertise au service des interrogations postcoloniales. Nous en avons pourtant besoin pour éviter les risques d'une lecture "postcoloniale » simpliste des images et de leurs errements dans le temps. Les artistes, en revanche, se sont souvent révélés plus perspicaces, en particulier dans des films comme Caché de Michael Haneke (2005), La Graine et le mulet d'Abdellatif Kechiche (2007 ; fig. 5) ou Un prophète de Jacques Audiard (2009), qui évoquent et retravaillent des histoires, des mémoires et des héritages de la colonisation et de la décolonisation avec des propos qui dépassent, par la force et les nuances de leurs images, à la fois la discussion publique et les travaux d'historiens et d'intellectuels.

Rémi Labrusse. Pour aborder la question du pouvoir, l'approche postcoloniale s'est emparée de notions auxquelles elle a donné un sens nouveau : celles d'" empire " et de "différence ", notamment. À partir de là, elle a repensé les questions de la violence et de l'identité, en dépassant le seul cadre historique du colonialisme occidental moderne. Sur cet horizon où l'histoire s'associe à l'anthropologie, en quoi un regard postcolonial peut-il conduire à considérer autrement la violence et les pulsions identitaires à l'œuvre dans toute production d'images?

Zahia Rahmani. Questionner l'apport des études postcoloniales aux théories de I'art, de la violence et de l'identité donne à ce champ une amplitude séduisante mais limite aussi les possibilités de le circonscrire. Par conséquent, cela peut conforter l'idée assez malveillante que ces recherches relèvent d'un " carnaval académique ${ }^{17}$, un défoulement sans réelle efficacité historique. Le postcolonial n'est pas l'étude par le monde académique occidental de l'autre ou de l'oubli de I'autre ; c'est d'abord la prise en charge par un monde non académique - celui des écrivains et des opposants politiques - de ce qui, par ignorance, incompétence, oubli, mépris ou absence de volonté a été écarté par la langue de l'empire. Des pans entiers de savoirs sont alors à mettre au jour et à défricher. Nombre d'études post- 


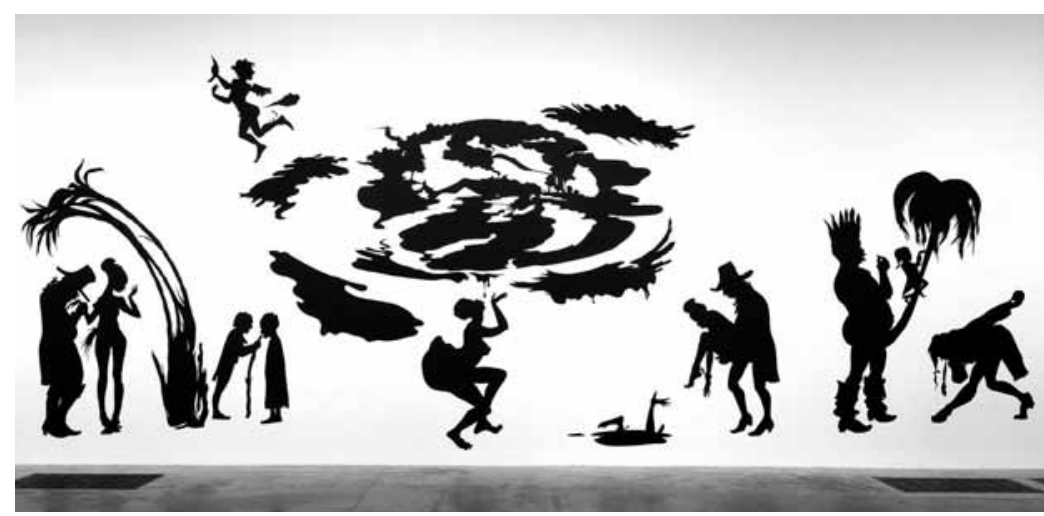

6. Kara Walker, Grub for Sharks: A Concession to the Negro Populace, Londres, Tate Modern, 2004. l'affront de la dévirilisation en situation d'homme assujetti ${ }^{19}$. Cette réflexion n'est pas étrangère au remarquable travail de l'artiste Kara Walker sur l'esclavage (fig. 6) ${ }^{20}$.

Si l'on part de l'idée que toute production d'image est travaillée par la violence des pulsions identitaires, il faut revoir tout l'édifice moderniste et lire aussi la tradition formaliste américaine sous cet angle. Barnett Newman ou Mark Rothko auraient participé d'une même conscience en prenant en charge la possibilité de perpétuer un art après l'effondrement que fut l'extermination du juif européen, et en fait de l'homme européen. Peut-on pour autant dire que ces œuvres relèvent d'une théorie postcoloniale? II ne me semble pas, et pourtant leurs œuvres, par leur qualité sublime, affectent le silence des sans voix. Selon l'approche postcoloniale, l'Europe, dans son histoire contemporaine, aurait instruit en son corps un sujet étranger à son identité matricielle, une figure de " paria », et la compréhension de cette exclusion se serait aussi effectuée par l'art. Selon cette logique, cette œuvre abstraite existe alors en réaction à la figuration, celle-ci étant devenue irreprésentable. Mes propos n'altèrent aucunement la qualité esthétique et critique des œuvres convoquées.

Maureen Murphy. Dans les pays anglo-saxons, les études postcoloniales ont introduit une forme de suspicion à l'égard de la construction du savoir passé mais également présent. Les archives, les œuvres et les images sont analysées à la lumière des rapports de pouvoir induits par la colonisation. II s'agit souvent de souligner ou du moins de ne pas passer sous silence l'héritage colonial, tout en donnant la parole aux agents représentés (ce systématisme frôlant parfois le normatif et le politiquement correct). En France, ces deux aspects de la démarche postcoloniale ont nourri de manière différenciée et parfois polémique ${ }^{21}$ les réflexions universitaires des dix dernières années, l'ironie du sort voulant en outre que les textes fondateurs des études postcoloniales relèvent en partie de la littérature française. Tandis que I'histoire de l'art s'ouvre lentement aux questions de pouvoir, de domination coloniale et plus largement de politique et de société ${ }^{22}$, les musées peinent à rendre publics de tels questionnements. Au Musée du quai Branly par exemple, inauguré en 2006 et dont les collections sont en lien direct avec l'histoire coloniale puisqu'elles sont, pour la majorité, issues de contrées anciennement colonisées, cette histoire n'est pas abordée dans le parcours permanent. Elle n'est questionnée que dans le cadre d'expositions temporaires ou de colloques. De même, le point de vue des auteurs des objets exposés et la question du présent de la création dans les pays concernés sont évoqués de manière marginale et ponctuelle. 
L'exposition Exhibitions : I'invention du sauvage en 2011 témoigne de ce report de l'institution sur des partenaires extérieurs pour porter une histoire chargée et douloureuse (fig. 7$)^{23}$. Or, les commissaires invités, Lilian Thuram et Pascal Blanchard, se positionnent plutôt du côté du mémoriel que de l'histoire. Ce choix est assez symptomatique de la situation française, où le besoin d'histoire, faute d'être suffisamment porté par les institutions, trouve un écho dans les milieux associatifs ou militants dont I'approche est plutôt empathique qu'analytique et n'est pas toujours propice à une compréhension nuancée. Dans l'exposition, sont mis sur le même plan les représentations de monstres et de coloniaux, une association, qui même si elle a pu être opérée ponctuellement dans le passé, était loin d'être systématique. Peu de place est faite à la complexité des moments historiques, aux débats (rien n'est dit, par exemple, des mouvements anti-coloniaux qui ont dénoncé le type d'exhibition en question) ou même à l'identité des personnes exhibées. Faute d'analyse permettant de comprendre les ressorts des constructions imaginaires dans le temps, I'exposition procède presque à l'inverse de l'objectif qu'elle s'était fixé et réactive des stéréotypes, plutôt que de donner les outils de leur déconstruction ou du moins de leur compréhension.

Todd Shepard. Au sein du débat naissant autour de l'histoire coloniale en France, les travaux majeurs qui dévoilent et mettent en avant des histoires de violence ont malheureusement souvent été compris comme l'unique optique permettant de réfléchir à la pertinence actuelle des références à la période coloniale. Comme I'historien américain Joshua Cole le remarque à propos des travaux historiques récents portant sur la guerre d'Algérie, " un consensus tacite veut que toutes les questions les plus controversées à propos de ce que le passé colonial de la France signifie pour la société française contemporaine - la possibilité d'intégrer les musulmans dans la vie politique, le sens de la citoyenneté nationale dans un monde postcolonial, le traitement des individus originaires d'Afrique du Nord par la police et les autorités - renvoient en dernier ressort à une scène primitive instituée par les actes de torture perpétrés pendant les années de la guerre [d'Algérie] ${ }^{24}$. Si les travaux sur la violence "coloniale » n'en demeurent pas moins importants, ils sont parfois un obstacle qui empêche de prendre la pleine mesure de notre monde postcolonial.

S'intéresser uniquement à la violence physique peut alimenter une histoire dualiste, qui présente le régime colonial comme contradictoire avec les valeurs républicaines et donc inévitablement promis à la faillite. Bien des travaux sur l'impérialisme républicain font grand cas des contradictions entre ces valeurs - liberté, égalité, fraternité ; les droits de l'Homme ; la démocratie ; la laïcité - et les mécanismes de la colonisation. C'est une histoire rassurante, en ce qu'elle suggère que l'empire colonial de la République n'était qu'une erreur ou une déviation, et que sa fin ou son échec était le résultat de valeurs et de méthodes véritablement républicaines qui se trouvaient désormais restaurées et confirmées. Et pourtant toutes les Républiques, de la Première à la Cinquième, ont joui d'un empire colonial. Accroître et renforcer l'assise du pouvoir dans les colonies était essentiel pour la construction des institutions républicaines en métropole ; très peu de républicains étaient anticolonialistes, tandis que nombre d'opposants au colonialisme en métropole étaient antirépublicains. Les penseurs du postcolonialisme réclament donc que nous nous intéressions au rôle constitutif de l'empire dans la démocratie républicaine et libérale et dans la France contemporaine. Lorsque nous nous tournons vers l'histoire de l'art, nous devons étudier, comme le note Daniel J. Sherman, comment des figures comme Paul Gauguin ou Jean Dubuffet ont pu critiquer vivement les prétentions occidentales à la supériorité

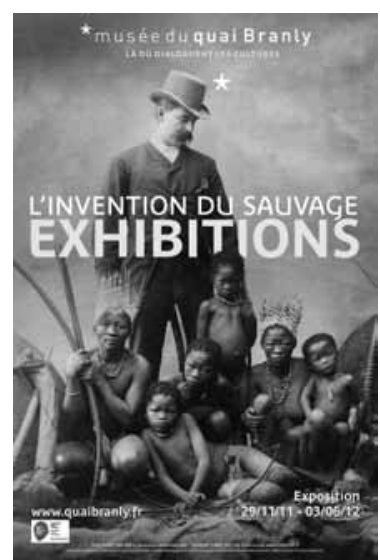

7. Affiche de l'exposition Exhibitions : I'invention du sauvage, Paris, Musée du quai Branly, 2011-2012. 
- esthétique, morale et politique - par rapport aux cultures qualifiées de " primitives ", alors que les deux hommes ont pu se consacrer pleinement à leur art au sein de réseaux et à travers des institutions qui soutenaient activement la colonisation ${ }^{25}$. S'intéresser avec une attention égale à leur critique de l'empire colonial et à leur collaboration avec celui-ci conduit à dépasser l'idée selon laquelle, en raison de valeurs universelles, l'empire colonial était voué à s'éteindre ; cette histoire plus trouble nous permet aussi de tenter de penser la longévité de l'empire colonial et la pérennité de son héritage.

Elvan Zabunyan. Penser la question du pouvoir par le biais d'une approche postcoloniale qui se serait "emparée » des notions d'empire et de différence ne me semble pas la meilleure manière d'aborder notre problématique. N'oublions pas en effet que le siècle des Lumières est aussi celui de l'esclavage et de la colonisation. Dès lors, une disjonction s'opère entre la construction d'une modernité « éclairée » et la mise en place d'un système de répression politique et économique dont les conséquences sont proportionnellement aussi dévastatrices pour les individus asservis qu'elles sont bénéfiques pour les pays occidentaux se constituant en puissances impériales. Il est par conséquent difficile d'appliquer à ce constat des analyses généralistes en établissant des liens unilatéraux de cause à effet, notamment lorsqu'il s'agit d'étudier les notions d'identité coloniale et postcoloniale selon une orientation déterminée par la question de la violence comme conséquence et non comme raison. Dans «De la violence", le titre du premier chapitre des Damnés de la Terre de Frantz Fanon, publié en 1961, une phrase très éclairante nous permet de contredire cette idée de " pulsions identitaires » qui évoque une forme de pathologie agressive au détriment d'une construction structurée de l'identité culturelle : "La décolonisation ne passe jamais inaperçue car elle porte sur l'être, elle modifie fondamentalement l'être, elle transforme des spectateurs écrasés d'inessentialité en acteurs privilégiés, saisis de façon quasi grandiose par le faisceau de l'Histoire. Elle introduit dans l'être un rythme propre, apporté par les nouveaux hommes, un nouveau langage, une nouvelle humanité. [...] La « chose » colonisée devient homme dans le processus même par lequel elle se libère ${ }^{26}$. C'est sur ce genre d'affirmation que la pensée postcoloniale a pu s'établir comme vecteur d'une réflexion plurielle permettant de convoquer la production aussi bien de textes que d'images. Comme le souligne Stuart Hall dans son analyse de l'œuvre de Fanon, il s'agit d'accorder toute son importance à la question du " regard "; elle qui est au cœur de la fonction représentative, qui édifie les structures propres du champ des humanités, et qui permet de penser le sujet et sa subjectivité ${ }^{27}$.

Rémi Labrusse. Face à l'ordre des discours, au pouvoir des langages institutionnels, peut-on parler d'un principe de désordre, assumé comme tel et propre à la démarche postcoloniale, lorsque s'y mêlent l'analyse critique, l'engagement politique et la création esthétique? Quelles interactions concrètes en résultent, aujourd'hui, entre le champ des sciences humaines et les mondes de l'art? Dans ces interactions, n'observe-t-on pas une sorte de friction paradoxale - et en elle-même productive - entre adhésion et déception, entre élan et mélancolie?

Todd Shepard. Les débats postcoloniaux sont d'abord le fait de penseurs qui se sont intéressés à la situation de certaines anciennes colonies après la période des indépendances, enlisées dans leurs déceptions quant à leurs attentes non réalisées et quant à la forme qu'auraient dû assumer la politique et la réflexion. Ces érudits, Gayatri 
Spivak, Homi Bhaba, Michel-Rolph Trouillot, Gyan Prakash et David Scott s'interrogeaient : comment était-il possible d'entériner les apports des penseurs anticolonialistes tout en reconnaissant que les victoires des activistes anticolonialistes s'étaient avérées moins fécondes qu'ils ne l'avaient imaginé ? De quelle manière les limites des progrès postérieurs à l'indépendance influencent-elles les tentatives d'analyse des défaites de l'anticolonialisme - tout en insistant dans le même temps sur la pérennité de l'empire en tant que référence pertinente pour comprendre le passé et le présent ?

Leur réaction a surtout consisté à rejeter les manichéismes de toutes sortes, de ceux qui nient l'humanité des colonisés comme de ceux qui exigent que la quête d'authenticité assimile ces réactions au colonialisme et à ses avatars. La complexification et le rejet des systèmes binaires ont donc été un élément essentiel dans les efforts des penseurs du postcolonialisme pour mener une réflexion qui intègre les impasses et les limites de la pensée anticoloniale. La critique postcoloniale implique hybridité et complexité.

Cet engagement a eu pour résultat bien connu des textes denses et difficiles. La capacité à dénoncer des arrangements confortables en est un autre. Dans le sillage des célèbres travaux sur les systèmes réalisés par l'artiste Hans Haacke (fig. 8), qui ont révélé les relations profondes entre le monde de l'art et le soutien apporté à l'exploitation capitaliste, ou l'apartheid en Afrique du Sud, entre autres ${ }^{28}$, des travaux historiques sur les musées de France - du Musée des arts africains et océaniens au début des années 1960 au Musée du quai Branly plus récemment, mais aussi au Louvre ont révélé les efforts faits pour effacer tout lien avec l'époque coloniale ${ }^{29}$. Au-delà de la condamnation pure et simple, ce qui est intéressant dans ces travaux, c'est que les histoires simples de rôle positif ou négatif, refusant les complications perturbatrices, échouent à montrer l'utilité de l'empire, mais aussi de sa disparition.

Elvan Zabunyan. En considérant les axes de recherche qui réfléchissent, à partir de méthodologies distinctes mais conjointes, à des possibilités de décloisonner les catégories constituées souvent par pur confort académique, le terme de " désordre " est souvent évoqué. Cela fut le cas pour les cultural studies initiées dès 1964 à l'université de Birmingham, ainsi que pour le département de History of Consciousness ouvert en 1968 à l'université de Santa Cruz en Californie. Les théories et études postcoloniales ne sont pas épargnées par cette définition à double sens : la notion de désordre peut être comprise comme un affront à l'ordre établi et une dilution des repères traditionnels, mais aussi comme un changement, une révolution, un mouvement. Paradoxalement, en les définissant par des termes imprécis (" flottement», etc.), on cherche à les disqualifier et à minimiser leur importance, alors qu'il s'agit du tâtonnement habituel de la recherche qui est ici comme partout décelable. La simple revendication d'une posture qui assume une union de forces disciplinaires s'avère, selon les points de vue, aussi salvatrice que suicidaire. Au-delà d'un champ unique et repéré, les études postcoloniales ou culturelles s'accordent la liberté de provoquer des rencontres entre les spécialités comme l’histoire, la littérature, la géopolitique ou les arts visuels pour utiliser les outils propres à celles-ci tout en en dégageant des orientations inédites qui soulignent l'importance de constituer un réseau de pensées critiques. La Triennale Intense Proximité inau-
8. Hans Haacke, Shapolsky et al., Manhattan Real-Estate Holdings, a Real-Time Social System, as of May 1, 1971, 1971, Paris, Musée national d'Art moderne.

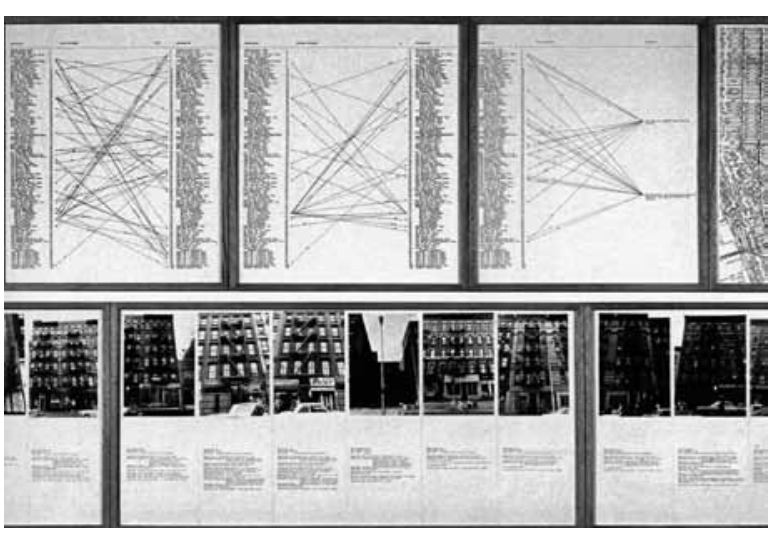


9. David Adjaye, Philip Freelon, maquette du National Museum of African American History and Culture au National Mall à Washington, D.C., ouverture du musée prévue en 2015.

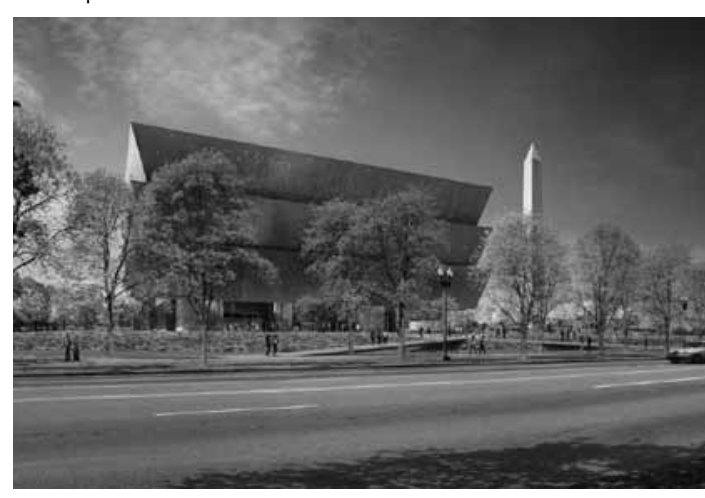

gurée au Palais de Tokyo à Paris en avril 2012 sous la direction artistique d'Okwui Enwezor et l'anthologie qui l'accompagne s'ancrent très nettement dans ce réseau ${ }^{30}$.

$C^{\prime}$ est au sein de cette richesse que les liens tissés entre les sciences humaines et le monde de l'art doivent être compris. Si l'on considère plus spécifiquement les interactions dans le domaine de l'art contemporain, les analyses les plus concrètes sont convoquées grâce aux expositions et aux publications qui les accompagnent. Ainsi, pour en citer quelques-unes, les problématiques portant sur les notions d'altérité, de différence, de pouvoir, de migration, d'information, d'image sontelles récurrentes dans les manifestations internationales organisées aujourd'hui par les musées. En cela, il n'y a pas lieu de considérer comme menaçant l'apport des théories postcoloniales comme si celles-ci allaient venir détrôner l'hégémonie d'une histoire de l'art occidental enseignée en France. Si un véritable travail de fond est mené sur leur contenu et sur la généalogie de leur existence, elles permettent de contextualiser I'histoire mondiale de ces trois derniers siècles en proposant de revoir nos points de vue, comme le suggérait Said. Le troisième chapitre de son ouvrage Culture et impérialisme revient sur cette question : "Nous sommes tenus de prendre en compte la disparité durable du rapport de forces entre l'Occident et le nonOccident si nous voulons comprendre avec précision des formes culturelles comme le roman, les discours ethnographiques et historiques, une certaine poésie et un certain opéra, où abondent les allusions à cette disparité et les structures fondées sur elle ${ }^{31}$. En transposant cette affirmation aux arts visuels, on saisit que c'est par le lien que ces derniers peuvent tisser avec les théories postcoloniales que s'impose une histoire de regards permettant de renverser les rapports de forces.

Maureen Murphy. Ce n'est pas un hasard si les études postcoloniales sont nées dans des pays anglo-saxons à tradition multiculturaliste, tels que l'Angleterre, les ÉtatsUnis et l'Australie. Le rapport particulier entretenu dans ces pays avec les différentes communautés qui les constituent a fourni un terrain favorable à l'épanouissement des études postcoloniales, des études subalternes ou des études sur le genre. Développées dans le champ académique, ces réflexions ont rapidement dépassé les frontières universitaires pour participer aux débats de société qui animaient ces pays dans les années 1970 et 1980. Les musées eux-mêmes ont pris en compte cette attention portée à la voix des " opprimés » et l'ont intégrée à leur politique. Si l'on considère par exemple le Mall à Washington, parc bordé de grands musées fédéraux qui tentent, par leur pluralité, de circonscrire une certaine image de la nation américaine, on se rend compte que chaque minorité y est représentée, le dernier projet prévu étant celui des Afro-américains, qui ouvrira en 2015 (fig. 9).

Aussi louables que soient ces projets, ils n'en posent pas moins la question de la légitimité de la parole. Au National Museum of the American Indian, par exemple, n'est entendue que celle des Indiens, comme si le fait d'être Indien conférait une plus grande légitimité au contenu énoncé. Le critère identitaire semble parfois l'emporter sur la pertinence du propos. Tout se passe comme si les rapports de pouvoir s'étaient renversés sur un plan symbolique, de l'ordre du postulat éthique, et non sur un plan réel. Nous sommes loin du désordre introduit dans les années 1970 et vivons aujourd'hui une forme de sclérose de la pensée qui semble parfois privilégier (symboliquement) la voix des opprimés par rapport à la polyphonie et au dialogue. 
En France, ces débats n'ont pas véritablement émergé, l'une des raisons étant sans doute l'attachement du pays à l'idéal unitaire républicain. Un travail de mémoire et de prise de conscience s'avère pourtant nécessaire, si l'on ne souhaite pas voir violemment ressurgir les souffrances du passé. Ce décalage temporel entre la France et les pays anglo-saxons est à mon avis une chance, au sens où cela n'induit pas, aujourd'hui, de déconstruire chacune des disciplines, mais plutôt d'y introduire une once de questionnement critique salvateur et constructif.

Zahia Rahmani. Pour que les études postcoloniales voient le jour, une certaine indiscipline a été nécessaire. Mais certains principes n'en sont pas exclus pour autant. Un fondement éthique leur est inhérent; il en est à la fois la limite et la garantie. Il consiste à faire valoir un principe de déconstruction du " site » et de déplacement du regard. Le mot " site » est ici à prendre dans un sens élargi ; le tissu, par exemple le chintz ou le wax, peut être étudié sous cet angle, selon une méthode postcoloniale. On ne cesse de citer l'artiste Yinka Shonibare et son utilisation du wax comme référent à l'histoire de l'Afrique et de l'esclavage, mais on omet de dire que le tissu " anglais » dont il dépouille ses personnages pour le remplacer par du wax, est un coton imprimé moghol, prisé par les classes moyennes puis par la bourgeoisie et par l'aristocratie du XVIII ${ }^{e}$ siècle (fig. 3). Seuls les Indiens maîtrisaient cette technique. Le goût anglais pour ces cotonnades peintes (dont le chintz, un tissu pour l'ameublement) fit la fortune, entre autres, de la Compagnie des Indes britanniques. Elle a imposé son pouvoir à I'Inde, l'a dépossédée de son savoir-faire et a ruiné son économie. Recouvrir ce tissu par le wax, c'est aussi révéler une histoire pour en masquer une autre.

De manière plus générale, les études postcoloniales ont aussi vu le jour parce que, comme le dit Said, un certain nombre de gens, bien sous tout rapport mais fatigués de rester au seuil de la porte indéfiniment, ont fini un jour par la forcer ${ }^{32}$. Si une certaine forme de mélancolie de l'empire subsiste, c'est que la promesse qu'il portait n'a pas été tenue. Des sociétés dépossédées de leur patrimoine ne peuvent sans déception assumer ce présent commun si l'égalité devant le patrimoine $\mathrm{n}^{\prime}$ est pas effective. C'est ce que Mbembe, citant Gandhi, nomme le triple mouvement : I'ellipse, le décrochage et la reprise. Cette situation de reprise ne nous permet pas de porter un regard formel sur les œuvres des artistes en prise avec l'identité postcoloniale. La critique qui en est faite relève toujours du commentaire anthropologique. Le signifiant "local» domine. Dans ce cas, l'esthétique n'est pas uniquement reléguée, elle est volontairement ignorée. Les commissaires d'expositions sont eux-mêmes responsables de cet état de fait. Mais peuvent-ils faire autrement ? Et en ont-ils les moyens ? Il est donc nécessaire d'évaluer la manière dont le "biographique » a contaminé l'art. Par exemple, on s'interdit de mettre en parallèle l'œuvre de I'artiste conceptuel Joseph Kosuth One and Three chairs, datée de 1965, avec la reprise qu'en a faite Jumana Emil Abboud dans son triptyque vidéo An Untitled LifeDrawing, réalisé en 2006 (fig. 10). On ne voit dans ce 10a. Joseph Kosuth, One and Three Chairs, 1965, Paris, Musée national d'art moderne ; b. Jumana Emil Abboud, An Untitled Life-Drawing, 2006 (http://vimeo.com/34772774).
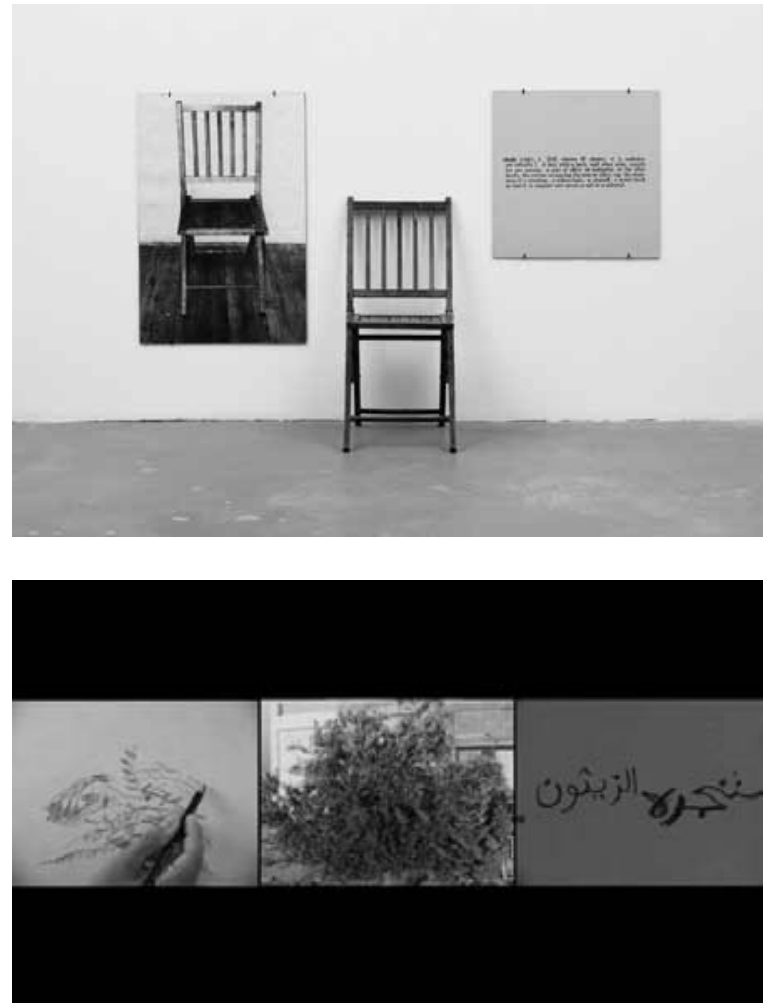
travail que le présent d'une Palestinienne, alors qu'en réactivant l'œuvre de Kosuth, elle lui confère un potentiel critique qui n'avait jamais jusqu'alors été révélé. Dans le contexte des études postcoloniales, les œuvres d'art contemporain sont de vrais activateurs pour penser de nouvelles méthodes critiques en histoire de l'art. Mais encore faut-il le voir et le vouloir. Les études postcoloniales participent d'une transition nécessaire et même urgente, mais en aucun cas elles ne doivent faire l'économie d'une analyse qui consiste à penser ce qu'elles ont dû longtemps abandonner pour être opérantes. Ces études, qui ont relégué le marxisme comme une pensée de I'« ethnocentre», ont dû, avec la mondialisation, le réintégrer comme composante de sa critique. Il est bon de rappeler que la critique esthétique et formelle, et cela a pu se justifier, n'a jamais été le point fort des études postcoloniales, les obligeant à repenser ces catégories.

Nota bene : ce texte résulte d'un échange de courriels et fait suite au débat qui a eu lieu à l'Institut national d'histoire de l'art le 16 janvier 2012.

La contribution de Todd Shepard a été traduite par Géraldine Bretault.

1. Edward Said, Orientalism, New York, 1978 [éd. fr. : L'Orientalisme : l'Orient créé par l'Occident, Paris, 2005].

2. Voir notamment Bill Ashcroft, Gareth Griffiths, Helen Tiffin éd., The Post-Colonial Studies Reader, Londres/New York, 1995 ; Ania Loomba éd., Colonialism/Postcolonialism, Londres/New York, 1998 ; Bill Ashcroft, Gareth Griffiths, Helen Tiffin éd., Post-Colonial Studies: The Key Concepts, Londres/ New York, 2001 ; Reina Lewis, Sara Mills éd., Feminist Postcolonial Theory, Édimbourg, 2003. Il est à noter que ces ouvrages n'ont pas d'entrées « arts visuels ». Ce sont avant tout les catalogues publiés à l'occasion de manifestations artistiques internationales qui prennent le relais en la matière. Voir par exemple Unpacking Europe: Towards a Critical Reading, Salah Hassan, Iftikhar Dadi éd., (cat. expo., Rotterdam, Museum Boijmans Van Beuningen, 2001), Rotterdam, 2011, ou les publications des cinq plateformes de la Documenta 11 en 2002 sous la direction d'Okwui Enwezor.

3. Magiciens de la terre, Jean-Hubert Martin éd., (cat. expo., Paris, Musée national d'art moderneCentre Georges Pompidou/La grande halle de la Villette, 1989), Paris, 1989.

4. 'Primitivism' in $20^{\text {th }}$ Century Art: Affinity of the Tribal and the Modern, William Rubin éd., (cat. expo., New York, Museum of Modern Art/Detroit, Detroit Institute of Arts, 1984), New York/Boston, 1984.

5. Voir entre autres Yve-Alain Bois, "La Pensée sauvage ", dans Art in America, 73, avril 1985, p. 178-189 ; Thomas McEvilley, "Doctor, Lawyer, Indian Chief: 'Primitivism' in $19^{\text {th }}$ Century Art at the
Museum of Modern Art in 1984 ", dans Art Forum, 23/3, novembre 1984, p. 54-61; James Clifford, " Histories of the Tribal and the Modern ", dans Art in America, 73, avril 1985, p. 164-174.

6. Voir Gayatri Spivak, In Other Worlds: Essays in Cultural Politics, New York, 1987.

7. Said, 1978 (2005), cité n. 1.

8. Cette bibliographie réalisée dans le cadre du programme "L'art dans la mondialisation » est consultable via le site de I'INHA : www.inha.fr/spip. php?rubrique399.

9. The Short Century: Independence and Liberation Movements in Africa, 1945-1994, (cat. expo., New York, Museum of Modern Art, 2002), New York, 2002.

10. Ce collectif animé par Ranajit Guha a réuni, entre autres, Dipesh Chakrabarty, Partha Chatterjee et Gayatri Spivak.

11. Les douze volumes regroupés sous le titre Subaltern Studies Series sont un corpus unique et rare dans I'histoire de la recherche contemporaine; ils ont rendu possible une connaissance autre et nouvelle de l'empire, alliant un constat sur le présent et une étude de la relation de l'effet à la cause. Pour comprendre la radicalité et la nouveauté de la critique sociale de ces chercheurs, voir Gautam Bhadra, Gyanendra Pandey, "From Mughal to British ", dans Ranajit Guha, Gayatri Spivak éd., Subaltern Studies, Delhi, 1988.

12. Éric Michaud, Histoire de l'art : une discipline à ses frontières, Paris, 2005.

13. Eric Hobsbawm, Terence Ranger éd., L'Invention de la tradition, Paris, 2006 (2012) [éd. orig. : The Invention of Tradition, Cambridge, 1983]. 
14. Jacques Derrida, "La mythologie blanche", dans Marges de la philosophie, Paris, 1972, p. 323.

15. Voir Jean-Loup Amselle, L'Art de la friche : essai sur l'art africain contemporain, Paris, 2005.

16. Citons les travaux de Rémi Labrusse sur les collections d'art islamique en France, Islamophilies: I'Europe moderne et les arts de I'Islam, Paris, 2011.

17. Voir Jean-François Bayard, Les Études postcoloniales : un carnaval académique, Paris, 2010.

18. Achille Mbembe est un intellectuel d'origine camerounaise qui travaille surtout sur l'Afrique contemporaine et qui écrit en direction des Africains. Cet intellectuel brillant et engagé, que I'on range de ce fait du côté des subversifs, a fait ses études en France avant d'enseigner aux États-Unis puis de devenir directeur de recherches dans un institut de sciences sociales en Afrique du Sud.

19. Achille Mbembe, "Le potentat sexuel. À propos de la sodomie, de la fellation et autres privautés postcoloniales ", dans Le Messager, 13 février 2006, publié en ligne: www.africultures.com/php/index. php?nav $=$ article $\&$ no $=4296$.

20. Voir l'exposition Kara Walker, My Complement, My Enemy, My Oppressor, My Love, (cat. expo., Minneapolis, Walker Art Center/New York, Whitney Museum of American Art/Los Angeles, UCLA Hammer Museum, 2007-2008), Minneapolis, 2007.

21. Voir Marie-Claude Smouts éd., La Situation postcoloniale: les Postcolonial Studies dans le débat français, Paris, 2007.

22. Laurence Bertrand Dorléac est l'une des actrices essentielles de cette ouverture de la discipline aux questions de société et de politique. II n'est pas anodin qu'elle dirige aujourd'hui la chaire d'histoire de I'art et de politique de Sciences Po Paris, créée en 2009.

23. Exhibitions: l'invention du sauvage, Pascal Blanchard, Gilles Boëtsch, Nanette Jacomijn Snoep éd., (cat. expo., Paris, Musée du quai Branly, 20112012), Paris, 2011

24. « ... a tacit consensus holds that all of the most controversial questions about what France's colonial past means for contemporary French society - the possibility of integrating Muslims into the polity, the meaning of national citizenship in a postcolonial world, the treatment of people of North African descent by police and other government officials refer ultimately back to a primal scene established by acts of torture during the [Algerian] war years »
(Joshua Cole, «Intimate Acts and Unspeakable Relations: Remembering Torture and the War for Algerian Independence ", dans Alec G. Hargreaves éd., Memory, Empire and Postcolonialism: Legacies of French Colonialism, Lanham, 2005, p. 125-141).

25. Daniel J. Sherman, French Primitivism and the Ends of Empire, 1945-1975, Chicago, 2011.

26. Frantz Fanon, Les Damnés de la terre, Paris, 1961, p. 66-67.

27. Voir notamment Stuart Hall, "The After-life of Frantz Fanon: Why Fanon? Why Now? Why Black Skin, White Mask? ", dans The Fact of Blackness: Frantz Fanon and Visual Representation, Londres, 1995, p. 12-37. Cette publication a été réalisée à la suite du colloque organisé à l'occasion de l'exposition Mirage: Enigmas of Race, Difference and Desire, (cat. expo., Londres, Institute of Contemporary Arts, 1995), Londres, 1995. Les artistes invités (Sonia Boyce, Renée Green, Lyle Ashton Harris, Isaac Julien, Marc Latamie, Glenn Ligon, Steve McQueen...), inscrits dans une réflexion sur la représentation de la figure noire (britannique ou américaine), convoquaient de façon privilégiée la pensée de Frantz Fanon dans leur processus de création visuelle.

28. Voir notamment les œuvres de Hans Haacke : Shapolsky et al. Manhattan Real Estate Holdings, A Real Time Social System, as of May 1, 1971 (1971, Paris, Musée national d'art moderne, Centre Georges Pompidou), et A Breed Apart (1978, Londres, Tate Modern). Sur ce sujet, voir aussi Pierre Bourdieu, Hans Haacke, Libre-Échange, Paris, 1994.

29. Dominic Thomas, "The Quai Branly Museum Political Transition, Memory and Globalisation in Contemporary France », dans French Cultural Studies, 19/2, juin 2008, p. 141-157 ; Sherman, 2011, cité n. 25.

30. Okwui Enwezor éd., Intense proximité : une anthologie du proche et du lointain, Paris, 2012.

31. Edward Said, Culture et impérialisme, Paris, 2000, p. 277 [éd. orig. : Culture and Imperialism, New York, 1993].

32. Edward Said, «Représenter le colonisé. Les interlocuteurs de l'anthropologie", dans Réflexions sur l'exil et autres essais, Arles, 2008, p. 391.

Mots-clés
colonialisme, empire, muséologie,
théories postcoloniales, transculturalisme

